\title{
Interference Management via Rate Splitting and HARQ over Time-Varying Fading Channels
}

\author{
Marco Levorato \\ DEI, University of Padova \\ Padova, Italy \\ levorato@dei.unipd.it
}

\author{
Osvaldo Simeone \\ Center for Wireless Comm. \\ and Signal Processing, NJIT \\ Newark, NJ, USA \\ osvaldo.simeone@njit.edu
}

\author{
Urbashi Mitra \\ Dept. of Electrical \\ Engineering, USC \\ Los Angeles, CA, USA \\ ubli@usc.edu
}

\begin{abstract}
The coexistence of two unlicensed links is considered, where one link interferes with the transmission of the other, over a timevarying, block-fading channel. In the absence of fading, standard information-theoretic results for the scenario at hand prove the sumrate optimality of single-user detection or joint decoding at the interfered receiver, depending on the instantaneous value of the interference power. These results hinge critically on the presence of full channel state information at some of the nodes. In this paper, the problem is revisited with quasi-static fading and in the absence of channel state information at the nodes by assuming that: (i) HARQ type-I is used at both links; (ii) the channels exhibit Markovian memory over the time-slots; (iii) ACK/ NACK messages of the two HARQ processes are received at the interfering transmitter and used to adapt the current access strategy and transmission parameters. The problem is formulated as a Partially Observable Markov Decision Process (POMDP) and a greedy solution is proposed. Numerical results highlight, on the one hand, the differences between the optimal design with or without channel state information and, on the other, the advantages of exploiting channel memory for interference management.
\end{abstract}

\section{Categories and Subject Descriptors}

C.2.1 [Computer-Communication Networks]: Network Architecture and Design—network communications, wireless communication

\section{General Terms}

Management

\section{Keywords}

Interference Management, Correlated Fading Channels, Cognitive Networks, Feedback-based Network Control

\section{INTRODUCTION}

As the number of wireless devices in the unlicensed band increases, novel decentralized interference management techniques

Permission to make digital or hard copies of all or part of this work for personal or classroom use is granted without fee provided that copies are not made or distributed for profit or commercial advantage and that copies bear this notice and the full citation on the first page. To copy otherwise, to republish, to post on servers or to redistribute to lists, requires prior specific permission and/or a fee.

CoRoNet'09, September 21, 2009, Beijing, China.

Copyright 2009 ACM 978-1-60558-738-7/09/09 ...\$10.00. become necessary to provide acceptable quality-of-service (see, e.g., [1]). The currently deployed solutions to this problem are, either interference avoidance (in the form of carrier-sense strategies or frequency hopping [2]) or underlay transmission (e.g., for UWB). There has been interest in interference management for some time (e.g. [3]); this work and recent results (e.g. [4]) point to the gains to be achieved via sophisticated signal processing wherein interfering links are allowed to transmit simultaneously, and joint decoding of the useful signal and part of the interference is carried out at the interfered receivers. We assume two interfering links, for simplicity, for the rest of this paper. The most general transmission strategy is the so-called rate-splitting scheme (see, e.g., [4]), where interfering transmitters split their information bits (or message) into two parts: The first, referred to as private, is decoded only at the intended destination, while the second, the common part, is decoded at both the intended destination and the interfered link. The goal of this strategy is to facilitate the interfered receiver, by allowing the latter to exploit the codebook structure of the interference (more precisely, of the common part) when decoding.

Information-theoretic results typically assume fixed and known channels, where the Channel State Information (CSI) is available at all nodes. Conclusive results have been found only for scenarios where the interference power is either stronger or weaker than the direct (useful) signal. In particular, it has been shown that with strong interference, transmission of only common information is optimal, since the interfered receiver can decode anything that the intended receiver can, while for weak interference only private information should be transmitted and treated as noise at the interfered receivers (see [4] for a review and further results) ${ }^{1}$. Rather than assuming fixed and known channels, a two-link interfering channel with ergodic fading (i.e., channels vary in an ergodic fashion along any transmitted packet) and full (non-causal) CSI at all nodes was recently studied in [5]. Interestingly, the latter paper shows that, if the users are given the possibility to allocate their power over multiple channel states, there is little performance loss in terms of sum-rate by transmitting only private information and only when the interference is sufficiently small. In other words, under the stated assumptions, rate-splitting and common information transmission are not necessary.

In this paper, we focus on a two-link interference channel over quasi-static fading channels in the absence of a priori CSI at the transmitters. We focus on a scenario where one link (say, longrange) interferes with the other (say, short-range), but not vice versa, as shown in Fig. 1-(a). Such scenario applies for instance to a cellular downlink overlaid with a femtocell, where the base

\footnotetext{
${ }^{1}$ Optimality should be intended in terms of the entire region of achievable rates for strong interference and only in terms of sumrate for weak interference.
} 
station (transmitter 2) communicates with a user (receiver 2) and interferes reception of a home base station (receiver 1) in the femtocell (transmitter 1 is a home user of the femtocell). A second scenario of interest is a cognitive system where the secondary user (transmitter 2) interferes with a primary receiver (receiver 1), while negligible interference is created by the primary system to the secondary (e.g., the secondary receiver knows the primary message a priori similar to [Devroye] and can thus cancel the interference). The model also applies with minor modifications to the case where the signal from the primary is treated as noise at the secondary receiver (and hence only increases the background noise)

The model in Fig. 1 is also referred to as Z-interference channel, which has been widely studied in the information-theoretic literature [6-9], see Fig. 1-(b). The two links employ HARQ type-I, i.e., undecoded packets are retransmitted and decoding takes place only based on the last received packet. The fading channels are assumed to vary according to Markov processes, whose statistics are known to all nodes. The problem we tackle is that of optimizing the access and transmission strategy of transmitter 2, i.e., of the interfering (long-range) link so as to maximize the overall system throughput.

The basic ideas is that, using the observation of ACK and NACK messages issued by the two receivers and the history of previously made choices, the interfering transmitter can infer the interference it is currently creating and thus adapt its strategy accordingly. The idea follows previous work $[11,12]$ where observation of ACK/ NACK messages was used to adapt the transmission rate over a point-to-point quasi-static channel (see also [13] for related work). Adaptive secondary access and power control strategies for a cognitive radio system in which the primary (interfered) user, unlike our model, always treats secondary signal as noise are investigated in [14].

\section{SYSTEM MODEL}

Consider the two coexisting links of Fig. 1-(a). Transmitter 2 's signal interferes with the intended signal for receiver 1 . The two transmitters are symbol and frame synchronous and operate in blocks of $n$ channel uses each. The received signals at the two receiver in each channel use $i=1, \ldots, n$ and block $b$ are given by, respectively:

$$
\begin{aligned}
& Y_{1, i, b}=h_{1, b} X_{1, i, b}+\sqrt{\alpha} h_{21, b} X_{2, i, b}+Z_{1, i, b} \\
& Y_{2, i, b}=h_{2, b} X_{2, i, b}+Z_{2, i, b},
\end{aligned}
$$

where we assume per-block power constraints $1 / n \sum_{i=1}^{n}\left|X_{j, i, b}\right|^{2} \leq P_{j}$ for the two transmitters $j=1,2$, and unit-power white circularly symmetric Gaussian noises $Z_{1, i, b}$ and $Z_{2, i, b}$. Quasi-static fading channels are denoted by $h_{1, b}, h_{2, b}$, $h_{21, b}$ and are assumed to be independent, constant within each block and to follow Rayleigh fading. Equivalently, the channel gains $g_{1, b}=\left|h_{1, b}\right|^{2}, g_{2, b}=\left|h_{2, b}\right|^{2}, g_{21, b}=\left|h_{21, b}\right|^{2}$ are independent and exponentially distributed random variables with unit power. Variation of the fading channels across different blocks is modelled via Markov processes so that the joint distribution in adjacent slots is as in (2) at the bottom of the page [15], where $\rho$ is the temporal correlation coefficient and $\mathbf{I}_{0}$ is the Bessel function of the first kind and order zero. The fading channel realizations $g_{1}, g_{2}, g_{21}$ are unknown to the transmitters, but their statistics are known. Perfect receiver channel state information is assumed. Finally, the parameter $\alpha \geq 0$ defines the average power of the interference.

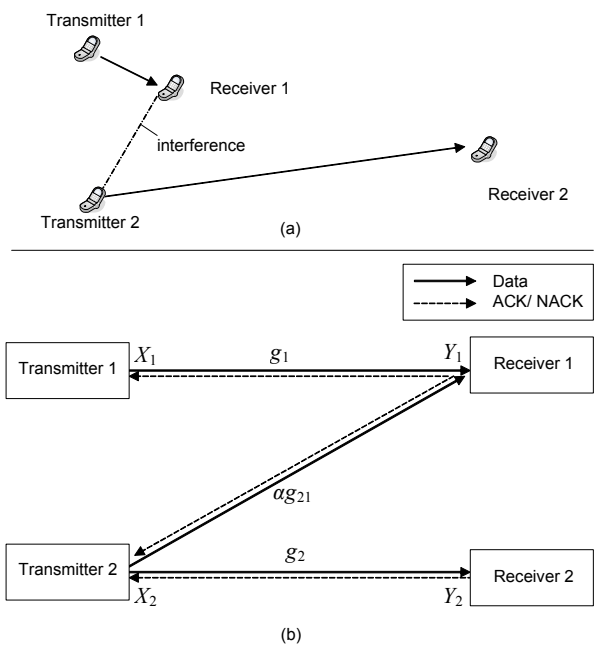

Figure 1: Two unlicensed links, the first short-range and the second long-range, coexist over the same bandwidth. The longrange transmission interferes the short-range link receiver; (b) The scenario above can be modelled as a Z-interference channel, which is studied here in the presence of quasi-static fading channels and HARQ transmission.

Transmitters are assumed to be always backlogged. Block length $n$ is assumed to be large enough for good codes to behave close to the theoretical limits. Due to quasi-static fading, outage events may occur at either receiver, and retransmissions in the form of HARQ type-I are implemented to combat this impairment. We assume that transmitter 1 transmits in every block, and employs a (Gaussian) codebook with fixed rate $R_{1}$ [bits/ channel use] and power $P_{1}$, where transmissions may be copies of previously transmitted packets given the use of HARQ. Transmitter 2 adapts its access and transmission strategy in order to better manage interference to receiver 1 . This is done by exploiting the information collected from the previously received ACK/ NACK messages issued by the receivers, and accounting for the strategies attempted in the previous slots (see Fig. 1-(b)).

\subsection{Interference Management via HARQ and Rate Splitting}

We define all the information available at transmitter 2 at time-slot $b$ as $\mathcal{I}_{b}$. This includes the history of previously received ACK/ NACK messages $\mathbf{e}_{b-1}=\left[e_{b-1}, e_{b-2}, \ldots, e_{1}\right]$ from the two receivers and the vector of the previously attempted strategies $\mathbf{u}_{b-1}=\left[u_{b-1}, u_{b-2}, \ldots, u_{1}\right]$. The feedback history includes the ACK/NACK sent by receiver 1 and receiver 2 , and thus $e_{b}=\left(f_{1, b}, f_{2, b}\right)$, where $f_{i, b}=0$ and $f_{i, b}=1$ means that receiver $i$ sent a NACK and an ACK in slot $b$, respectively. The strategy $u_{b}=\left(a_{b}, r_{b}, p_{b}\right) \in\{0,1\} \times[0,1] \times[0,1]$ is defined as follows. Based on $\mathcal{I}_{b}$, transmitter 2 may decide to: $(i)$ be silent, which corresponds to setting the activity variable to $a_{b}=0$; (ii) or transmit $\left(a_{b}=1\right)$ using a standard rate-splitting (Gaussian) superposition scheme characterized by the parameter pair $\left(r_{b}, p_{b}\right)$. In the latter case, transmitter 2 splits its rate into $r_{b} R_{2}$ (private part) and $\left(1-r_{b}\right) R_{2}$ (common part) with $r_{b} \in[0,1]$. Power is corre-

$$
\phi\left(\left|h_{c, b}\right|,\left|h_{c, b-1}\right|\right)=\frac{4\left|h_{c, b}\right|\left|h_{c, b-1}\right|}{1-\rho} \mathbf{I}_{0}\left(\frac{2 \sqrt{\rho}\left|h_{c, b}\right|\left|h_{c, b-1}\right|}{1-\rho}\right) e^{-\frac{\left.\left|h_{c, b}^{2}+\right| h_{c, b-1}\right|^{2}}{1-\rho}}
$$


spondingly split as $p_{b} P_{2}$ and $\left(1-p_{b}\right) P_{2}$ with $p_{b} \in[0,1]$. The goal is to maximize the average aggregate throughput in a window of $T$ slots. It is noted that, once a decision on $u_{b}=\left(a_{b}, r_{b}, p_{b}\right)$ is made at transmitter 2 , this has to be forwarded to receivers 1 and 2 .

The reliable rate regions can be described following standard information-theoretic arguments (see, e.g., [16]). If transmitter 2 is silent, then Receiver 1 successfully decodes the signal sent by transmitter 1 if $R_{1} \leq \mathcal{C}\left(g_{1} P_{1}\right)$ where $\mathcal{C}(x)=\log (1+x)$, is the Shannon capacity function. If transmitter 2 transmits, then the condition for successful decoding (assuming optimal joint decoding of useful signal and transmitter 2's common part) becomes

$$
\begin{gathered}
R_{1} \leq \mathcal{C}\left(\frac{g_{1, b} P_{1}}{1+\alpha g_{12, b} p_{b} P_{2}}\right) \\
R_{1}+\left(1-r_{b}\right) R_{2} \leq \mathcal{C}\left(\frac{g_{1, b} P_{1}+\alpha g_{12, b}\left(1-p_{b}\right) P_{2}}{1+\alpha g_{12 . b} p_{b} P_{2}}\right) .
\end{gathered}
$$

Receiver 2 instead successfully decodes the signal from transmitter 2 (assuming optimal joint decoding of private and common parts) if the following holds:

$$
r_{b} R_{2} \leq \mathcal{C}\left(g_{2, b} p_{b} P_{2}\right), \quad R_{2} \leq \mathcal{C}\left(g_{2, b} P_{2}\right) .
$$

Now, define the channel vector as $\gamma_{b}=\left[\begin{array}{lll}g_{1, b} & g_{2, b} & g_{21, b}\end{array}\right]$ and the throughput (bits/sec/Hz) for block $b$ as

$$
G\left(u_{b}, \gamma_{b}\right)=R_{1} s_{1}\left(u_{b}, \gamma_{b}\right)+R_{2} s_{2}\left(u_{b}, \gamma_{b}\right),
$$

where $s_{j}\left(u_{b}, \gamma_{b}\right)$ equals one if a packet for link $j=1,2$ is successfully delivered in slot $b$ and zero otherwise. Those functions can be derived from the previously shown decoding regions as follows. Define $1\{\cdot\}$ as the indicator function, we have:

$$
\begin{aligned}
& s_{1}\left(u_{b}, \gamma_{b}\right)=1\left\{\left(R_{1}, R_{2}\right) \in \mathcal{S}_{1}\left(u_{b}, \gamma_{b}\right)\right\} \\
& s_{2}\left(u_{b}, \gamma_{b}\right)=1\left\{R_{2}: R_{2} \in \mathcal{S}_{2}\left(u_{b}, \gamma_{b}\right)\right\},
\end{aligned}
$$

where $\mathcal{S}_{1}\left(u_{b}, \gamma_{b}\right)=\left\{\left(R_{1}, R_{2}\right): \quad R_{1} \leq \mathcal{C}\left(g_{1} P_{1}\right)\right\} \quad$ if $a_{b}=0$ and $\mathcal{S}_{1}\left(u_{b}, \gamma_{b}\right)=\left\{\left(R_{1}, R_{2}\right)\right.$ : (3a) is satisfied $\}$ if $a_{b}=1$. Moreover,

$$
\mathcal{S}_{2}\left(u_{b}, \gamma_{b}\right)=\left\{R_{2}: \text { (4) is satisfied }\right\} \text {. }
$$

The optimization of the average throughput over a window of $T$ slots amounts to choosing the mapping between the information $\mathcal{I}_{b}$ and the strategy $u_{b}$, denoted as $u_{b}\left(\mathcal{I}_{b}\right)$, for every $b=1, \ldots, T$ so that

$$
\max _{\left\{u_{b}\left(\mathcal{I}_{b}\right)\right\}_{b=1}^{T}} \sum_{b=1}^{T} E\left[G\left(u_{b}, \gamma_{b}\right)\right]
$$

where the average is taken with respect to the channel distribution. Denoting the optimal strategy as $u_{b}^{*}\left(\mathcal{I}_{b}\right)$, the problem above is equivalent to solving, for every block $b$,

$$
u_{b}^{*}\left(\mathcal{I}_{b}\right)=\arg \max _{u_{b}\left(\mathcal{I}_{b}\right)} E\left[G\left(u_{b}, \gamma_{b}\right)+\sum_{b^{\prime}=b+1}^{T} G\left(u_{b^{\prime}}^{*}, \gamma_{b^{\prime}}\right) \mid \mathcal{I}_{b}\right] .
$$

The problem stated in (9)-(10) is a Partially Observable Markov Decision Processes (POMDP).

\section{MEMORYLESS CHANNELS}

Let us consider at first the case where channels vary i.i.d. over the retransmission slots (i.e., $\rho=0$ in (2)). In this case, the available information $\mathcal{I}_{b}$ at transmitter 2 is not useful to adapt the transmission strategy, since it does not provide any information on the current channels' realization. The optimization in (10) then becomes

$$
u_{b}^{*}\left(\mathcal{I}_{b}\right)=\arg \max _{u_{b}} E\left[G\left(u_{b}, \gamma_{b}\right)\right] \text { for every } \mathcal{I}_{b},
$$

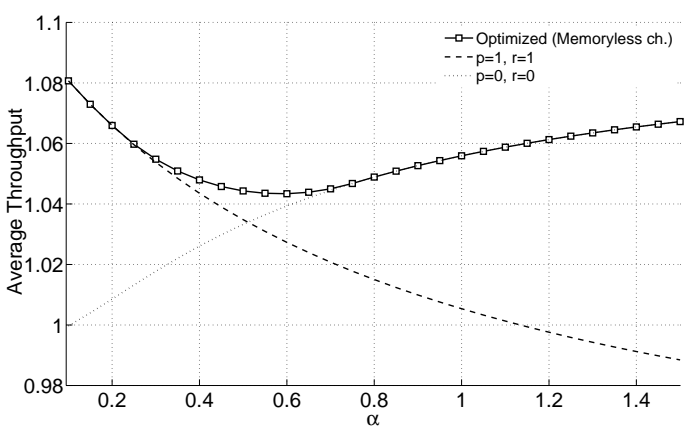

Figure 2: Average throughput achieved by the optimized policy and $\left(p_{b}, r_{b}\right)$ equal to $(1,1)$ (only private information) and $(0,0)$ (only common information), with $\beta_{1}=0.1$ and $\beta_{2}=0.9$.

which only depends on the marginal channel statistics. Therefore, it can be seen that the best transmission strategy for transmitter 2 is either to set $a_{b}=0$ (no transmission) for all $b$, which leads to the throughput

$$
\begin{aligned}
E\left[G\left(u_{b}, \gamma_{b}\right)\right]_{a_{b}=0} & =R_{1} \operatorname{Pr}\left\{\mathcal{S}_{1}\left(u_{b}, \gamma_{b}\right)\right\}_{a_{b}=0} \\
& =R_{1} \exp \left(-\left(2^{R_{1}}-1\right) / P_{1}\right),
\end{aligned}
$$

or to set $a_{b}=1$ with the rate-splitting parameters $\left(r_{b}, p_{b}\right)$ that maximize

$$
\begin{aligned}
E\left[G\left(u_{b}, \gamma_{b}\right)\right]_{a_{b}=1} & =R_{1} \operatorname{Pr}\left\{\mathcal{S}_{1}\left(u_{b}, \gamma_{b}\right)\right\}_{a_{b}=1}+ \\
& +R_{2} \operatorname{Pr}\left\{\mathcal{S}_{2}\left(u_{b}, \gamma_{b}\right)\right\}_{a_{b}=1},
\end{aligned}
$$

where $\operatorname{Pr}\left\{\mathcal{S}_{1}\left(u_{b}, \gamma_{b}\right)\right\}_{a_{b}=1}$ appears to be intractable and is thus determined numerically from $3 \mathrm{a}$ and

$$
\operatorname{Pr}\left\{\mathcal{S}_{2}\right\}_{a_{b}=1}= \begin{cases}1-\exp \left(-\max \left(\frac{{ }^{R_{2}-1}}{P_{2}}, \frac{2^{r_{b} R_{2}-1}}{p_{b} P_{2}}\right)\right) & \text { if } p_{b}>0 \\ 1-\exp \left(-\left(\frac{2 R_{2}-1}{P_{2}}\right)\right) & \text { if } r_{b}=0, p_{b}=0 \\ 1 & \text { if } r_{b}>0, p_{b}=0\end{cases}
$$

It can be seen that the success probability (15) for the second link is minimized for both $p_{b}=r_{b}=1$ (only private information) and $p_{b}=r_{b}=0$ (only common information), that is, when no rate-splitting is performed. Instead, the outage probability $\operatorname{Pr}\left\{\mathcal{S}_{1}\left(u_{b}, \gamma_{b}\right)\right\}_{a_{b}=1}$ for the first link is minimized if $p_{b}=0$ and $r_{b}=1$, where the latter condition corresponds to the extreme case where the second transmitters uses full power for a common message of zero rate. From the above, it is concluded that there is a trade-off between optimizing the two outage probabilities with respect to the transmission strategy of the second link. We are interested in the optimal trade-off with respect to the average throughput (9), which is studied next via numerical results.

\subsection{Numerical Results}

Let us fix the transmission rates of transmitter 1 and 2 to a fraction $\beta_{1}$ and $\beta_{2}$ of their respective capacities with neither interference nor fading, i.e., $R_{1}=\beta_{1} \mathcal{C}\left(P_{1}\right)$ and $R_{2}=\beta_{2} \mathcal{C}\left(P_{2}\right)$. Fig. 2 compares the average throughput achieved by the optimized policy with that achieved when $\left(p_{b}, r_{b}\right)$ is equal to $(1,1)$ (only private information) and $(0,0)$ (only common information) as a function of the interference channel gain $\alpha$, for $\beta_{1}=0.1, \beta_{2}=0.9$ and $P_{1}=P_{2}=4$. Fig. 3 depicts the optimal values of $p_{b}$ and $r_{b}$ for $\beta_{2}=0.9$ and $\beta_{1}=0.1,0.3,0.5$ and 0.9 as a function of $\alpha$.

It can be observed that when $\alpha$ is small, the optimal strategy is to transmit the private part only, i.e., $(p, r)=(1,1)$. Conversely, when $\alpha$ is sufficiently large the optimal strategy is to transmit common 


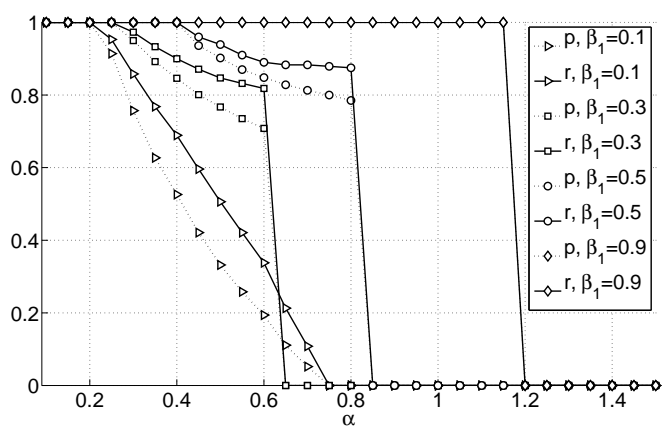

Figure 3: Optimal values of $p$ and $r$ as a function of $\alpha, \beta_{2}=0.9$ and $\beta_{1}=0.1,0.3$ and 0.5 .

part only, i.e., $(p, r)=(0,0)$. This is consistent with well-known results for the sum-rate capacity of non-fading Gaussian interference channels (see, e.g., [8]), where the sum-rate-optimal solution switches from all-private to all-common at $\alpha=1$. However, in our scenario, due to fading and the absence of CSI, we do not have a hard threshold, but rather a gradual switching between these two strategies, and furthermore, the switching tends to occur for $\alpha<1$. However, as $\beta_{1}$ is increased in Fig. 3, the switching point tends to increase, i.e., it takes a larger value of the interference gain $\alpha$ to make transmission of only common information optimal.

Related results have been recently presented in the parallel work [17] (see also references therein) for the diversity-multiplexing trade-off of an interference channel.

\section{CORRELATED CHANNELS}

In the previous Section, we addressed the optimization of the transmission strategy of transmitter 2 under the assumption of memoryless channels $(\rho=0)$. In the following, we consider timecorrelated channels $(\rho \geq 0)$. In this case, the information conveyed by $\mathcal{I}_{b}=\left\{\mathbf{u}_{b-1}, \mathbf{e}_{b-1}\right\}$, i.e., the decisions and the feedback (ACK, NACK) associated with the previous time slots, can be exploited in order to improve the average throughput, Eq. 9), achieved in the next slots. Intuitively, prior decisions and feedback identify a sequence of possible regions for the previously realized channel gains $\left\{\gamma_{b-1}, \ldots, \gamma_{1}\right\}$ that helps transmitter 2 set $u_{b}$ based on the a posteriori distribution of the current channel $\gamma_{b}$.

The solution of the optimization problem of Eq. (10) is intractable. Therefore, we address instead the following greedy solution

$$
\tilde{u}_{b}^{*}\left(\mathcal{I}_{b}\right)=\arg \max _{u_{b}\left(\mathcal{I}_{b}\right)} E\left[G\left(u_{b}, \gamma_{b}\right) \mid \mathcal{I}_{b}\right] .
$$

Thus, transmitter 2, when selecting the action in a certain time slot, maximizes the expected average throughput in the next time slot, disregarding the long-term future evolution of the transmission process. It is important to recall that transmitter 2 selects an action at the beginning of each time slot, based on the available $\mathcal{I}_{b}$, and is thus able to follow the transmission process step-by-step. The main difference between the optimal and the greedy controller lies in the capability of the former to select actions while also considering the information that will be available in the following decision instants (i.e., the former allows more sophisticated exploration of the channel gain space).
For the memoryless case, we argued in the previous section via numerical results, that the optimal choice of $\left(p_{b}, r_{b}\right)$ is equal either to $(1,1)$ or $(0,0)$, apart from a small region of values of $\alpha$ (coinciding with the switching point between those two values). Therefore, in order to keep computational complexity low, we restrict the strategy space of transmitter 2 to $\left(p_{b}, r_{b}\right)=(0,0)$ or $\left(p_{b}, r_{b}\right)=(1,1)$. More precisely, the action space is reduced to three elements, namely

$$
u_{b}= \begin{cases}0 & \text { if } a_{b}=0 \\ 1 & \text { if } a_{b}=1, p_{b}=0, r_{b}=0 \\ 2 & \text { if } a_{b}=1, p_{b}=1, r_{b}=1\end{cases}
$$

Recalling that $\gamma_{b}=\left(g_{1, b}, g_{2, b}, g_{21, b}\right)$ we define the regions

$$
\overline{\mathcal{R}_{1}}(u)=\left\{\left(g_{1}, g_{21}\right):\left[R_{1}, R_{2}\right] \notin \mathcal{S}_{1}(u, \gamma)\right\} \subset \mathbb{R}^{2},
$$

where the dependence of the region $\overline{\mathcal{R}_{1}}(u)$ on $R_{1}$ and $R_{2}$ has been dropped for the sake of notational clarity. Thus, $\overline{\mathcal{R}_{1}}(u)$ is the region of channel gains $\left[g_{1}, g_{21}\right]$ where receiver 1 incurs failure conditioned on the control variable $u_{b}=u$. Analogously, we can define $\overline{\mathcal{R}_{2}}(u)=\left\{g_{2}:\left[R_{2}\right] \notin \mathcal{S}_{2}(u, \gamma)\right\} \subset \mathbb{R}$, for $u=1,2$ and $\overline{\mathcal{R}_{2}}(0)=\mathbb{R}$, as when $u=0$ then transmitter 2 does not transmit. We also denote with $\mathcal{R}_{1}(u)$ and $\mathcal{R}_{2}(u)$ the complementary regions of $\overline{\mathcal{R}_{1}}(u)$ and $\overline{\mathcal{R}_{2}}(u)$, respectively. Thus, $\mathcal{R}_{1}(u)$ and $\mathcal{R}_{2}(u)$ represent the region of channel gains where receiver 1 and receiver 2 decode successfully the intended signal. As an example of the type of information these regions may provide due to feedback, if $\left(g_{1, b}, g_{21, b}\right) \in \overline{\mathcal{R}_{1}}(0)$, receiver 1 fails to receive the packet sent by transmitter 1 in slot $b$ (and thus a NACK is issued) while transmitter 2 is idle: In this case, one can infer that $g_{1, b} \leq 2^{R_{1}}-1 / P_{1}$, while $g_{21, b}$ can take any value.

Eq. (16) can be written as follows

$$
\tilde{u}_{b}^{*}\left(\mathcal{I}_{b}\right)=\arg \max _{u_{b}} \int_{0}^{+\infty} G\left(u_{b}, \gamma_{b}\right) \phi\left(\gamma_{b} \mid \mathcal{I}_{b}\right) \mathrm{d} \gamma_{b},
$$

where $\phi(\cdot)$ represents the probability density functions (pdf) of the arguments. In order to make the decision that maximizes (19), transmitter 2 needs to know the distribution $\phi\left(\gamma_{b} \mid \mathcal{I}_{b}\right)$. This distribution can be split into two terms

$$
\phi\left(\gamma_{b} \mid \mathcal{I}_{b}\right)=\phi\left(g_{2, b} \mid \mathbf{u}_{b-1}, \mathbf{f}_{2, b-1}\right) \phi\left(\left(g_{1, b}, g_{21, b}\right) \mid \mathbf{u}_{b-1}, \mathbf{f}_{1, b-1}\right) .
$$

The distribution $\phi\left(\left(g_{1, b}, g_{21, b}\right) \mid \mathbf{u}_{b-1}, \mathbf{f}_{1, b-1}\right)$ can be obtained as in Eq. (21), where $\phi\left(g_{1, b} g_{21, b} \mid g_{1, b-1} g_{21, b-1}\right)$ can be derived from (2) and $\mathcal{R}_{1}\left(u_{b}, f_{1, b}\right)$ is equal to $\overline{\mathcal{R}_{1}}\left(u_{b}\right)$ and $\mathcal{R}_{1}\left(u_{b}\right)$ if $f_{1, b}=0$ and $f_{1, b}=1$, respectively. $\phi\left(g_{1,0} g_{21,0} \mid \mathbf{u}_{-1}, \mathbf{f}_{1,-1}\right)$ is initialized with the joint unconditioned distribution of $g_{1}$ and $g_{21}$. Thus, the distribution $\phi\left(\left(g_{1, b}, g_{21, b}\right) \mid \mathbf{u}_{b-1}, \mathbf{f}_{1, b-1}\right)$ is updated in each slot, according to the received feedback (ACK/NACK) from receiver 1 . For instance, if the received feedback in slot $b$ is an ACK, then transmitter 2 knows that the pair $\left(g_{1, b}, g_{21, b}\right)$ is in $\mathcal{R}\left(u_{b}, 1\right)$, and thus computes the a posteriori distribution of the channel gains for the next slot by integrating and normalizing the distribution over this region only. Analogously, we can compute $\phi\left(g_{2, b} \mid \mathbf{u}_{b-1}, \mathbf{f}_{2, b-1}\right)$ recursively as

$$
\frac{\int_{\mathcal{R}_{2}\left(u_{b}, f_{2, b}\right)} \phi\left(g_{2, b} \mid g_{2, b-1}\right) \phi\left(g_{2, b-1} \mid \mathbf{u}_{b-2}, \mathbf{f}_{2, b-2}\right) \mathrm{d} g_{2, b-1}}{\int_{R_{2}\left(u_{b}, f_{2, b}\right)} \phi\left(g_{2, b-1} \mid \mathbf{u}_{b-2}, \mathbf{f}_{2, b-2}\right) \mathrm{d} g_{2, b-1}}
$$

$$
\phi\left\{\left(g_{1, b}, g_{21, b}\right) \mid \mathbf{u}_{b-1}, \mathbf{f}_{1, b-1}\right\}=\frac{\iint_{\mathcal{R}_{1}\left(u_{b-1}, f_{1, b-1}\right)} \phi\left(g_{1, b} \mid g_{1, b-1}\right) \phi\left(g_{21, b} \mid g_{21, b-1}\right) \phi\left(g_{1, b-1} g_{21, b-1} \mid \mathbf{u}_{b-2}, \mathbf{f}_{1, b-2}\right) \mathrm{d} g_{1, b-1} \mathrm{~d} g_{21, b-1}}{\iint_{\mathcal{R}_{1}\left(u_{b-1}, f_{1, b-1}\right)} \phi\left(g_{1, b-1} g_{21, b-1} \mid \mathbf{u}_{b-2}, \mathbf{f}_{1, b-2}\right) \mathrm{d} g_{1, b-1} \mathrm{~d} g_{21, b-1}}
$$




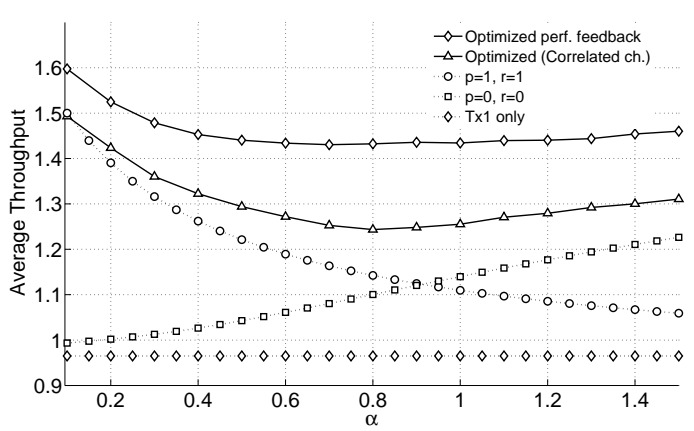

Figure 4: Average throughput achieved by the optimized policy, and by the policy where $u_{b}$ is kept fixed to 0 (transmitter 1 only), 1 and 2 as a function of $\alpha . \beta_{1}=0.7, \beta_{2}=0.9$ and $\rho=0.8$.

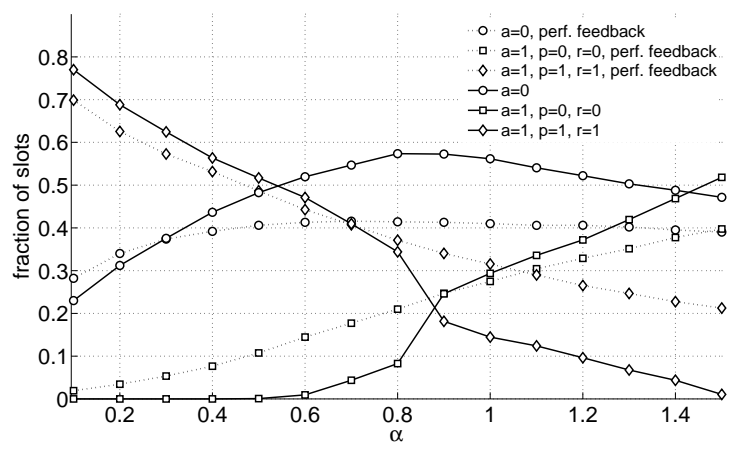

Figure 5: Fraction of slots in which $u_{b}^{*}$ takes value 0,1 and 2 as a function of $\alpha, \beta_{1}=0.7, \beta_{2}=0.9$ and $\rho=0.8$.

where $\mathcal{R}_{2}\left(u_{b}, f_{2, b}\right)$ is equal to $\overline{\mathcal{R}_{2}}\left(u_{b}\right)$ and $\mathcal{R}_{2}\left(u_{b}\right)$ if $f_{2, b}=0$ and $f_{2, b}=1$, respectively.

\subsection{Numerical Results}

In the following, we examine the average throughput achieved by the greedy-optimized policy for the correlated channel case, as compared to the average throughput of fixed policies in which $u_{b}$ is fixed to 0,1 and 2 for all $b$. Moreover, we show the average fraction of slots in which the greedy controller sets $u_{b}^{*}$ to 0,1 and 2. To obtain an upper bound, we also plot these metrics for the case where transmitter 2 has available the exact value of the gains associated with the three channels, i.e., $\gamma_{b}{ }^{2}$ We refer to this case as perfect feedback. It can be shown that with perfect feedback the optimal policy is greedy and can thus be computed similarly to the discussion above (see [12]).

Fig. 4 and 5 depict the metrics mentioned above as a function of $\alpha$ for $\beta_{1}=0.7, \beta_{2}=0.9$ and $\rho=0.8$. As expected, the greedy controller outperforms any fixed policy, by adapting the transmission policy to the actual channel conditions. It can be observed the strong dependence between the average interference gain $\alpha$ and the fraction of slots in which the controller selects the various transmission parameter: As in the memoryless case, when $\alpha$ is small the controller selects in most of the slots $p_{b}$ and $r_{b}$ equal to one (only private information). Nevertheless, in instances when transmitter 2 detects conditions in which its transmission would have poor success probability while generating significant interference to that of transmitter $1, u_{b}^{*}$ is set equal to zero, i.e., transmitter 2 keeps idle. This happends, for instance, if transmitter 2 receives a sequence of NACKs from receiver 1 associated to slots in which $u_{b}^{*}=2$, indicat-

\footnotetext{
${ }^{2}$ Thus the transmitter exactly knows $\gamma_{b}$ when selecting $u_{b+1}^{*}$
}

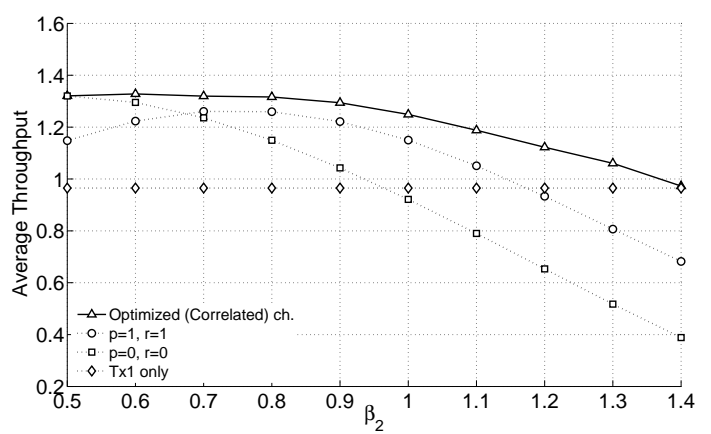

Figure 6: Average throughput achieved by the optimized policy, and by the policy where $u_{b}$ is kept fixed to 0 (transmitter 1 only), 1 and 2 as a function of $\beta_{2}$. $\beta_{1}=0.7, \alpha=0.5$ and $\rho=0.8$.

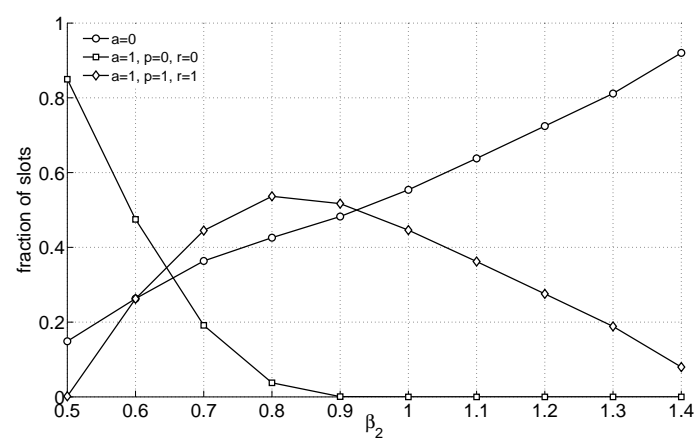

Figure 7: Fraction of slots in which $u_{b}^{*}$ takes value 0,1 and 2 as a function of $\beta_{2}$. $\beta_{1}=0.7, \alpha=0.5$ and $\rho=0.8$.

ing that the interfering channel is experiencing a fading coefficient sufficiently larger than that of the direct link between transmitter 1 (but not enough to let receiver 1 decode the signal from transmitter 2). Conversely, when $\alpha$ is large, then transmitter 2 selects in most of the slots $p_{b}$ and $r_{b}$ equal to 0 (only common information), in order to let receiver 1 decode and cancel the interfering channel. Again, in a significant fraction of slots transmitter 2 selects to keep idle due to the actual fading conditions. Similarly, it can be observed that when the controller is using both $p_{b}=r_{b}=1$ and $p_{b}=r_{b}=0$, i.e., when the value of $\alpha$ is in the region in which the interfering signal is treated as noise in some slots and decoded and cancelled in others, there is a significant fraction of slots in which transmitter 2 chooses to refrain from transmission. In fact, for those values of $\alpha g_{1, b}$ and $g_{21, b}$ have the highest probability of being in a region in which the interference signal has a received power large enough to generate significant interference if treated as noise, but not to be decoded by receiver one.

As shown in Fig. 4, with perfect feedback, the achieved throughput increases somewhat (around 6\% in this example). However, we underscore that perfect feedback entails that receiver 1 and 2 feed back real values, and receiver 1 feedback the gain of both the wanted and the interfering channel. In light of this, the performance loss of binary feedback with the proposed greedy strategy may be considered quite limited. Observing the optimal decisions corresponding to perfect feedback (Fig. 5), it can be seen that they are quite different from the binary feedback (ACK/NACK) case. This means that in the latter case the knowledge provided by the reception of ACK/NACK messages can be insufficient in some cases for making the optimal decision. It can also be observed that in the ACK/NACK feedback case, the average selection ratio of $u_{b}^{*}=1$ and $u_{b}^{*}=2$ has a sudden switch at a critical value of $\alpha$, while in the 


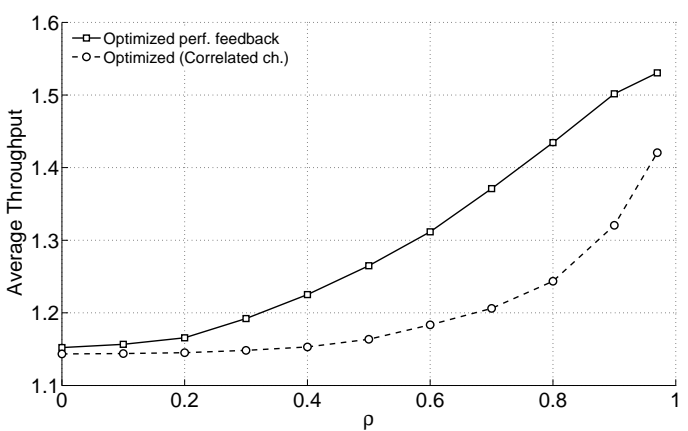

Figure 8: Average throughput for the perfect feedback and the optimized case

perfect feedback case, the selection ratio shows a softer transition, as the controller can better adapt its decision to the current state of the channel.

Fig. 6 and 7 show similar curves where $\alpha$ is fixed to 0.5 and $\beta_{2}$ is varied. As $\beta_{2}$ increases, the probability that transmitter 2 selects $p_{b}=r_{b}=0$ decreases, as the interfering signal at receiver 1 becomes more and more unlikely to be decoded. Interestingly, the probability that transmitter 2 selects $p_{b}=r_{b}=1$ first increases and then decreases, reaching a point of maximum close to $\beta_{2}=0.8$. In fact, while when $\beta_{2}$ is small it can be effectively decoded by receiver 1 , as $\beta_{2}$ becomes too large, then the best policy is more likely to be an idle transmitter 2, as it its signal may generate significant interference at receiver one, but the probability that it is decoded by receiver 2 gets smaller.

Channel correlation has a significant impact on the effectiveness of the controller, as illustrated in Fig. 8. This shows the average throughput as a function of the channel correlation $\rho$ along with the perfect-feedback upper bound. In the memoryless case $(\rho=0)$, the controller does not see any improvement of its prediction capabilities when collecting either the previous ACK/NACK messages or the exact channel values. Therefore, upper bound and binaryfeedback performance coincide. As $\rho$ is increased, the throughput achieved by the controller with perfect feedback increases until it reach a floor close to $\rho=1$. In the binary-feedback case, the controller needs a higher value of $\rho$ to significantly improve its performance with respect to the memoryless case. However, for $\rho$ sufficiently large, it can be seen that the knowledge provided by the ACK/NACK feedback yields a relatively small performance loss with respect to that of perfect feedback. In fact, in this case, at each further transmission with a different $u_{b}^{*}$, since the channel remains stable, the controller can potentially reduce the uncertainty region regarding the current channel conditions even with a binary feedback

\section{CONCLUDING REMARKS}

In this paper, we have studied the problem of interference management in the presence of quasi-static fading and no CSI by combining information-theoretic coding techniques with higher-layer control strategies based on HARQ type I. It has been demonstrated that, unlike the ergodic case with full CSI studied in [5], transmission of common information, and even more general forms of rate-splitting, may be (throughput) optimal with quasi-static fading and no CSI. Moreover, if the fading processes have memory over the transmission slots, interfering transmitters may predict the current value of the interference based on the received ACK/ NACK and react accordingly to better manage interference.

Interesting extensions include optimization over both ratesplitting parameters and transmission rates, which has the potential not only of better exploiting the current interference conditions but also of improving exploration of the channel space [12].

\section{REFERENCES}

[1] A. Jovicic, H. Wang, and P. Viswanath, "On network interference management," submitted to IEEE Trans. Inform. Theory (Mar. 2008).

[2] J.M. Peha, "Approaches to spectrum sharing," IEEE Comm. Mag., vol. 43, no. 2, pp. 10-12, Feb. 2005.

[3] Ahlswede, "The capacity region of a channel with two senders and two receivers," Ann. Prob., vol. 2, pp. 805-814, Oct. 1974.

[4] R. H. Etkin, D.N.C. Tse, H. Wang, "Gaussian interference channel capacity to within one bit," IEEE Trans. Inform. Theory, vol.54, no.12, pp.5534-5562, Dec. 2008.

[5] D. Tuninetti, "Gaussian fading interference channels: Power control," in Proc. Asilomar Conf. Signals, Systems and Computers, Oct. 2008.

[6] A. Carleial, "Interference channels," IEEE Trans. Inform. Theory, vol. 24, no. 1, pp. 60-70, Jan 1978.

[7] Te Han and K. Kobayashi, "A new achievable rate region for the interference channel," IEEE Trans. Inform. Theory, vol.27, no.1, pp. 49-60, Jan 1981.

[8] I. Sason, "On achievable rate regions for the Gaussian interference channel," IEEE Trans. Inform. Theory, vol. 50, no. 6, pp. 1345-1356, June 2004.

[9] G. Kramer, "Outer bounds on the capacity of Gaussian interference channels," IEEE Trans. Inform. Theory, vol. 50, no. 3, pp. 581-586, Mar. 2004.

[10] N. Devroye, P. Mitran and V. Tarokh, "Achievable rates in cognitive radio channels," IEEE Trans. Inform. Theory, vol. 52, no. 5, pp. 1813- 1827, May 2006.

[11] A. K. Karmokar, D.V. Djonin and V.K. Bhargava, "POMDP-based coding rate adaptation for type-I hybrid ARQ systems over fading channels with memory," IEEE Trans. Wireless Comm., vol. 5, no. 12, pp. 3512-3523, Dec. 2006.

[12] R. Aggarwal, P. Schniter, and C. E. Koksal, "Rate adaptation via link-layer feedback for goodput maximization over a time-varying channel," to appear in IEEE Trans. Wireless Commun [http://www.ece.osu.edu/ schniter/].

[13] Cong Shen, Tie Liu, M.P. Fitz, "Aggressive transmission with ARQ in quasi-static fading channels," in Proc. IEEE ICC 2008, pp. 1092-1097, May 2008.

[14] F. E. Lapicciriella, S. Huang, X. Liu and Z. Ding, "Feedback-based access and power control for distributed multiuser cognitive networks," in Proc. Info. Theory and Apps. Workshop, San Diego, CA, USA, Feb. 2009.

[15] C. C. Tan and C. Beaulieu, "On first-order markov modeling for the Rayleigh fading channel," IEEE Trans. Comm., vol. 48, no. 12, pp. 2032-2040, Dec. 2000.

[16] Chong Hon-Fah, M. Motani, H.K Garg, H. El Gamal, H., "On The Han-Kobayashi Region for theInterference Channel," IEEE Trans. Inform. Theory, vol. 54, no. 7, pp. 3188-3195, July 2008.

[17] Y. Weng, and D. Tuninetti, "Outage analysis of Block-Fading Gaussian Interference Channels," in Proc. IEEE Intern. Workshop on Signal Processing Advances in Wireless Communications (SPAWC 2009), Perugia, Italy, June, 2009. 